Canad. Math. Bull. Vol. 23 (2), 1980

\title{
MULTIFUNCTIONS AND INVERSE CLUSTER SETS
}

\author{
BY
}

JAMES E. JOSEPH

\begin{abstract}
In this paper the notion of inverse cluster set, which was recently introduced and studied for functions by T. R. Hamlett and P. E. Long (Proc. Amer. Math. Soc., 53 (1975), 470-476), is extended to and investigated for multifunctions. We generalize the notion of inverse cluster set, extend to multifunctions and generalize some known results for inverse cluster sets of functions and offer some new results. In the latter sections, compactness generalizations are characterized in terms of inverse cluster sets and some results on connected and conectivity functions are extended to multifunctions.
\end{abstract}

1. Introduction. In [3], Professors Hamlett and Long introduced and studied the notion of inverse cluster sets for functions. In their paper they obtained interesting results relating properties of inverse cluster sets for a function to the continuity properties of the function and to the properties of the graph of the function.

A multifunction from $a$ set $X$ to $a$ set $Y$ is a function from $X$ to $\mathscr{P}(Y)-\{\varnothing\}$, where $\mathscr{P}(Y)$ is the power set of $Y$. In this article we extend the notion of inverse cluster set to multifunctions, generalize the concept of inverse cluster set, extend to multifunctions and otherwise generalize some results from [3], generalize some results from [11] and present some results which are new for functions. General properties of inverse cluster sets for multifunctions are proved including necessary and sufficient conditions for multifunctions to satisfy certain continuity and graph conditions. In the later sections we employ inverse cluster sets to characterize regular, $\boldsymbol{m}$-compact, Lindelöf and $\boldsymbol{H}$-closed spaces and also extend to multifunctions a known theorem giving a condition under which a connected function is a connectivity function.

2. Some preliminaries. We will denote the closure of a subset $A$ of a topological space by $\mathrm{cl}(A)$, the collection of open neighborhoods of $A$ by $\Sigma(A)$ and the collection of closed neighborhoods of $A$ by $\Gamma(A)$. A subset $A$ of a space is a regular-open (regular-closed) subset of the space if $A$ is equal to the interior of its closure (closure of its interior). We will denote the family of

Received by the editors August 14, 1978 and, in revised form, February 27, 1979.

AMS (MOS) subject classifications (1970). Primary 54C60. Secondary 54C10, 54D15, 54D20, 54D25.

Key words and phrases. Multifunction, upper-semicontinuous, closed graph, inverse cluster set. 
regular-closed subsets which contain a subset $A$ of a space by $\nabla(A)$. The adherence of a filterbase $\Omega$ on a space will be denoted by $a d \Omega$. We will say that a point $x$ in a space is in the $\theta$-closure of a subset $A$ of the space $\left(x \in \operatorname{cl}_{\theta}(A)\right)$ if each $V \in \Gamma(x)$ satisfies $A \cap V \neq \varnothing$, that $A$ is $\theta$-closed if $\operatorname{cl}_{\theta}(A)=A$ and that $x$ is in the $\theta$-adherence of a filterbase $\Omega$ on the space $\left(x \in a d_{\theta} \Omega\right)$ if $x \in \operatorname{cl}_{\theta}(F)$ for each $F \in \Omega$ [14]. $A$ is $u$-closed if $A=\varnothing$ or $a d_{\theta} \Gamma(A)=A$.

We will represent the class of all multifunctions from a set $X$ to a set $Y$ by $\mathcal{M}(X, Y)$. Let $X$ and $Y$ be spaces and let $\alpha \in \mathcal{M}(X, Y)$. We say that $\alpha$ is upper-semicontinuous (u.s.c.) at $x \in X$ if for each $W \in \Sigma(\alpha(x))$ in $Y$ there is a $V \in \Sigma(x)$ in $X$ with $\alpha(V) \subset W$ (If $A \subset X$, then $\alpha(A)=\bigcup_{A} \alpha(x)$.); $\alpha$ is uppersemicontinuous on $X$ (u.s.c.) if $\alpha$ is u.s.c. at each $x \in X$. Smithson [10] has defined $\alpha$ to be weakly upper-semicontinuous (w.u.s.c.) at $x \in X$ if for each $W \in \Gamma(\alpha(x))$ there is a $V \in \Sigma(x)$ with $\alpha(V) \subset W$ and has called $\alpha$ weakly upper-semicontinuous on $X$ (w.u.s.c.) when $\alpha$ is w.u.s.c. at each $x \in X$. We note that if $\alpha$ is a function then $\alpha$ is w.u.s.c. at $x$ (w.u.s.c.) if and only if $\alpha$ is weakly-continuous at $x$ (weakly-continuous) in the sense of Levine [6]. We say

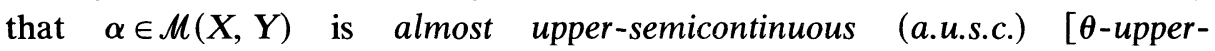
semicontinuous $(\theta$-u.s.c.)] at $x \in X$ if for each regular-open $W$ with $\alpha(x) \subset$ $W[W \in \Gamma(\alpha(x))]$ there is a $V \in \Sigma(x)[V \in \Gamma(x)]$ with $\alpha(V) \subset W$ and that $\alpha$ is

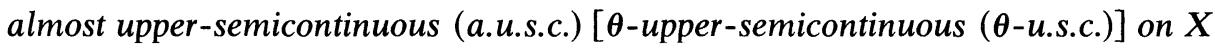
if $\alpha$ is a.u.s.c. [ $\theta$-u.s.c.] at each $x \in X ; \alpha$ has closed $(\theta$-closed) [compact] point images if $\alpha(x)$ is closed ( $\theta$-closed) [compact] in $Y$ for each $x \in X$. We will represent the graph of $\alpha$-i.e. $\{(x, y): x \in X$ and $y \in \alpha(x)\}$-by $\mathscr{G}(\alpha)$. As usual, we will say that $\alpha$ has a closed graph if $\mathscr{G}(\alpha)$ is a closed subset of $X \times Y$. Paralleling the notion of function with a strongly-closed graph from [4] we say that $\alpha$ has a strongly-closed graph if for each $(x, y) \in(X \times Y)-\mathscr{G}(\alpha)$ there are sets $V \in \Sigma(x)$, $W \in \Gamma(y)$, in $X$ and $Y$, respectively, with $(V \times W) \cap \mathscr{G}(\alpha)=\varnothing$. We say that $\alpha$ has a $\theta$-closed graph if $\mathscr{G}(\alpha)$ is a $\theta$-closed subset of $X \times Y$. In a product space $X \times Y$ the (2) $\theta$-closure of a subset $K\left((2) \operatorname{cl}_{\theta}(K)\right)$ of $X \times Y$ is $\{(x, y) \in X \times Y$ : all $V \in \Sigma(x)$ and $W \in \Gamma(y)$ satisfy $(V \times W) \cap K \neq \varnothing\} ; \quad K$ is (2) $\theta$-closed if (2) $\operatorname{cl}_{\theta}(K)=K$. It is not difficult to see that $\alpha \in M(X, Y)$ has a strongly-closed graph if and only if $\mathscr{G}(\alpha)$ is a (2) $\theta$-closed subset of $X \times Y$. Finally, if $X$ is a space, $x_{0} \in X$ and $\Omega$ is a filterbase on $X$ then $X$ with the topology $\{A \subset$ $X: x_{0} \notin A$ or $F \subset A$ for some $\left.F \in \Omega\right\}$ will be denoted by $X\left(x_{0}, \Omega\right)$. If $B \subset Y$, we denote $\{x \in X: \alpha(x) \cap B \neq \varnothing\}$ by $\alpha^{-1}(B)$.

3. Multifunctions, inverse cluster sets, graph and continuity properties. Let $X$ and $Y$ be spaces and let $\alpha \in M(X, Y)$. Employing equivalence (2) of Theorem 2.3 in [3] as a model we define the inverse cluster set of $\alpha$ at $y \in Y$ by $\mathscr{C}^{-1}(\alpha ; y)=\bigcap_{\Sigma(y)} \operatorname{cl}\left(\alpha^{-1}(V)\right)$. Generalizing this notion we define the strong inverse cluster set of $\alpha$ at $y \in Y$ to be $\mathscr{S}^{-1}(\alpha ; y)=\bigcap_{\Gamma(\mathrm{y})} \operatorname{cl}\left(\alpha^{-1}(V)\right)$, and the inverse $\theta$-cluster set of $\alpha$ at $y \in Y$ to be $\mathscr{T}^{-1}(\alpha ; y)=\bigcap_{\Gamma(y)} \operatorname{cl}_{\theta}\left(\alpha^{-1}(V)\right)$. In Lemma 3.1 we 
relate $\mathscr{C}^{-1}(\alpha ; y), \mathscr{S}^{-1}(\alpha ; y)$ and $\mathscr{T}^{-1}(\alpha ; y)$ to $\mathscr{G}(\alpha)$. We prove only statement (a) as the proofs of the other statements are similar; the projection from $X \times Y$ to $X$ is denoted by $\pi_{x}$.

3.1. Lemma. Let $X, Y$ be spaces and let $\alpha \in M(X, Y)$. Then

(a) $\mathscr{C}^{-1}(\alpha ; y)=\pi_{x}((X \times\{y\}) \cap c \ell(\mathscr{G}(\alpha)))$ for each $y \in Y$.

(b) $\mathscr{S}^{-1}(\alpha ; y)=\pi_{x}\left((X \times\{y\}) \cap(2) c \ell_{\theta}(\mathscr{G}(\alpha))\right)$ for each $y \in Y$.

(c) $\mathscr{T}^{-1}(\alpha ; y)=\pi_{x}\left((X \times\{y\}) \cap_{c} \ell_{\theta}(\mathscr{G}(\alpha))\right)$ for each $y \in Y$.

Proof of (a). Let $y \in Y, x \in \mathscr{C}^{-1}(\alpha ; y)$ and let $V \in \Sigma(x)$. Then for any $W \in$ $\Sigma(y)$ we have $V \cap \alpha^{-1}(W) \neq \varnothing$. Thus $(V \times W) \cap \mathscr{G}(\alpha) \neq \varnothing$. This shows that $(x, y) \in \operatorname{cl}(\mathscr{G}(\alpha))$ and, consequently, that $x \in \pi_{x}((X \times\{y\}) \cap \operatorname{cl}(\mathscr{G}(\alpha)))$. The steps in the above argument may be reversed to establish the reverse inclusion. The proof of (a) is complete.

In the first three theorems we characterize multifunctions with closed graphs, strongly-closed graphs and $\boldsymbol{\theta}$-closed graphs, respectively, in terms of inverse cluster sets. The proofs of Theorems 3.3 and 3.4 are similar to that of Theorem 3.2 and are omitted.

3.2. TheOREM. The following statements are equivalent for spaces $X, Y$ and $\alpha \in M(X, Y)$ :

(a) The multifunction $\alpha$ has a closed graph:

(b) $\alpha^{-1}(y)=\pi_{x}\left((X \times\{y\}) \cap_{c} \ell(\mathscr{G}(\alpha))\right)$ for each $y \in Y$.

(c) $\mathscr{C}^{-1}(\alpha ; y)=\alpha^{-1}(y)$ for each $y \in Y$.

Proof that (a) implies (b). Obvious.

Proof that (b) implies (c). This follows from Lemma 3.1(a).

Proof that (c) implies (a). Let $(x, y) \in(X \times Y)-\mathscr{G}(\alpha)$. Then $x \notin \alpha^{-1}(y)$, so $x \notin \mathscr{C}^{-1}(\alpha ; y)$. Thus there are sets $V \in \Sigma(x)$ in $X$ and $W \in \Sigma(y)$ in $Y$ with $V \cap \alpha^{-1}(W)=\varnothing$. This gives $(V \times W) \cap \mathscr{G}(\alpha)=\varnothing$.

This proof is complete.

3.3. TheOREM. The following statements are equivalent for spaces $X, Y$ and $\alpha \in M(X, Y)$ :

(a) The multifunction $\alpha$ has a strongly-closed graph;

(b) $\alpha^{-1}(y)=\pi_{x}\left((X \times\{y\}) \cap(2)_{c} \ell_{\theta}(\mathscr{G}(\alpha))\right)$ for each $y \in Y$.

(c) $\mathscr{S}^{-1}(\alpha ; y)=\alpha^{-1}(y)$ for each $y \in Y$.

3.4. TheOREM. The following statements are equivalent for spaces $X, Y$ and $\alpha \in M(X, Y)$ :

(a) The multifunction $\alpha$ has a $\theta$-closed graph;

(b) $\alpha^{-1}(y)=\pi_{x}\left((X \times\{y\}) \cap c \ell_{\theta}(\mathscr{G}(\alpha))\right)$ for each $y \in Y$.

(c) $\mathscr{T}^{-1}(\alpha ; y)=\alpha^{-1}(y)$ for each $y \in Y$.

We will next establish several results which relate inverse cluster sets and 
continuity properties. These results will include necessary and sufficient conditions for certain continuity properties in terms of inverse cluster sets. Let $X$ and $Y$ be spaces and let $\alpha \in M(X, Y)$. If $\Omega \subset \mathscr{P}(X)$ we write $\alpha(\Omega)=$ $\{\alpha(A): A \in \Omega\}$. We define the cluster set (strong cluster set) [ $\theta$-cluster set] of $\alpha \quad$ at $\quad x \in X \quad$ to be $\mathscr{C}(\alpha ; x)=a d \alpha(\Sigma(x)) \quad\left(\mathscr{S}(\alpha ; x)=a d_{\theta} \alpha(\Sigma(x))\right)$ $\left[\mathscr{T}(\alpha ; x)=a d_{\theta} \alpha(\Gamma(x))\right]$. The following relationships exist between cluster sets and inverse cluster sets. Again we prove statement (a) only.

3.5. Theorem. Let $X$ and $Y$ be spaces and let $\alpha \in M(X, Y)$ :

(a) $\mathscr{C}(\alpha ; x)=\left\{y \in Y: x \in \mathscr{C}^{-1}(\alpha ; y)\right\}$ for each $x \in X$.

(b) $\mathscr{S}(\alpha ; x)=\left\{y \in Y: x \in \mathscr{S}^{-1}(\alpha ; y)\right\}$ for each $x \in X$.

(c) $\mathscr{T}(\alpha ; x)=\left\{y \in Y: x \in \mathscr{T}^{-1}(\alpha ; y)\right\}$ for each $x \in X$.

Proof of (a). Let $y \in Y$ and let $x \in X$ with $y \in \mathscr{C}(\alpha ; x)\left[x \in \mathscr{C}^{-1}(\alpha ; y)\right]$. If $V \in \Sigma(x)$ in $X$ and $W \in \Sigma(y)$ in $Y$ then $\alpha(V) \cap W \neq \varnothing\left[V \cap \alpha^{-1}(W) \neq \varnothing\right]$; so we have $V \cap \alpha^{-1}(W) \neq \varnothing[\alpha(V) \cap W \neq \varnothing]$. The proof of (a) is complete.

The following theorem appears in [5].

3.6. Theorem. If $X$ and $Y$ are spaces and $\alpha \in M(X, Y)$ is u.s.c. at $x_{0} \in X$ then $\mathscr{S}\left(\alpha ; x_{0}\right)={ }_{c} \ell_{\theta}\left(\alpha\left(x_{0}\right)\right)$.

One of the main results of [3] (Theorem 2.9) shows that if $X$ and $Y$ are spaces with $Y$ compact Hausdorff then a function $\alpha: X \rightarrow Y$ is continuous at $x_{0} \in X$ if and only if $x_{0} \in \mathscr{C}^{-1}(\alpha ; y)$ for exactly one $y \in Y$. Theorem 3.7 generalizes this result and extends it to multifunctions.

3.7. Theorem. Let $X$ and $Y$ be spaces with $Y$ compact and let $\alpha \in M(X, y)$ have $\theta$-closed point images. Then $\alpha$ is u.s.c. at $x_{0} \in X$ if and only if $\mathscr{C}\left(\alpha ; x_{0}\right)=$ $\alpha\left(x_{0}\right)$.

Proof. Necessity. We see easily that $\alpha\left(x_{0}\right) \subset \mathscr{C}\left(\alpha ; x_{0}\right)$ and the reverse inclusion follows from Theorem 3.6 since $\mathscr{C}\left(\alpha ; x_{0}\right) \subset \mathscr{S}\left(\alpha ; x_{0}\right)=\operatorname{cl}_{\theta}\left(\alpha\left(x_{0}\right)\right)=\alpha\left(x_{0}\right)$.

Sufficiency. Assume that $W_{0} \in \Sigma\left(\alpha\left(x_{0}\right)\right)$ and that $\Omega=\left\{\alpha(V)-W_{0}: V \in\right.$ $\left.\Sigma\left(\alpha\left(x_{0}\right)\right)\right\}$ is a filterbase on $Y$. Since $Y$ is compact we have $\varnothing \neq a d \Omega=$ $\operatorname{ad} \alpha\left(\Sigma\left(x_{0}\right)\right)-W_{0}=\mathscr{C}\left(\alpha ; x_{0}\right)-W_{0}=\alpha\left(x_{0}\right)-W_{0}$. Thus $\alpha\left(x_{0}\right)-W_{0} \neq \varnothing$, a contradiction, and $\alpha$ is u.s.c. at $x_{0}$.

The proof of the theorem is complete.

In connection with our next three theorems, which are similar in nature to Theorem 3.7, we recall that a subset $K$ of a space $X$ is quasi $H$-closed relative to $X$ if for each cover $\Lambda$ of $K$ by open subsets of $X$ there is a finite $\Lambda^{*} \subset \Lambda$ with $K \subset \operatorname{cl}\left(\bigcup_{\Lambda^{*}} V\right)$. If $X$ is quasi $H$-closed relative to $X$ we say simply that $X$ is quasi $H$-closed [8]. A Hausdorff quasi $H$-closed space is called $H$-closed. Herrington [2] has shown that $K$ is quasi $H$-closed relative to a space $X$ if and only if each filterbase $\Omega$ on $K$ satisfies $K \cap a d_{\theta} \Omega \neq \varnothing$. 
3.8. Theorem. Let $X$ and $Y$ be spaces with $Y$ quasi $H$-closed and let $\alpha \in \mathcal{M}(X, Y)$ have $\theta$-closed point images. Then $\alpha$ is a.u.s.c. at $x_{0} \in X$ if and only if $\mathscr{S}\left(\alpha ; x_{0}\right)=\alpha\left(x_{0}\right)$.

Proof. Necessity. Clearly, $\alpha\left(x_{0}\right) \subset \mathscr{S}\left(\alpha ; x_{0}\right)$. On the other hand, if $y \notin \alpha\left(x_{0}\right)$ there is a $W \in \Sigma(y)$ with $\alpha\left(x_{0}\right) \subset Y-\operatorname{cl}(W)$. Since $Y-\operatorname{cl}(W)$ is regular-open and $\alpha$ is a.u.s.c. there is a $V \in \Sigma\left(x_{0}\right)$ with $\alpha(V) \subset Y-\operatorname{cl}(W)$. So $y \notin \mathscr{S}\left(\alpha ; x_{0}\right)$. Hence $\mathscr{S}\left(\alpha ; x_{0}\right)=\alpha\left(x_{0}\right)$.

Sufficiency. Assume that $W_{0}$ is a regular-open subset about $\alpha\left(x_{0}\right)$ and that $\Omega=\left\{\alpha(V)-W_{0}: V \in \Sigma(x)\right\}$ is a filterbase on $Y$. Then $\Omega$ is a filterbase on $Y-W_{0}$ which is regular-closed and, consequently, quasi $H$-closed relative to $X$. Thus $\varnothing \neq a d_{\theta} \Omega-W_{0} \subset \mathscr{S}\left(\alpha ; x_{0}\right)-W_{0}=\alpha\left(x_{0}\right)-W_{0}$. Thus $\alpha\left(x_{0}\right)-W_{0} \neq \varnothing$, a contradiction, and $\alpha$ is a.u.s.c. at $x_{0}$.

The proof of the theorem is complete.

If $X$ and $Y$ are spaces, an a.u.s.c. function $\alpha \in \mathcal{M}(X, Y)$ is called almost continuous in [13].

3.9. Corollary. Let $X$ and $Y$ be spaces with $Y$ H-closed. Then a function $\alpha \in \mathcal{M}(X, Y)$ is almost continuous at $x_{0} \in X$ if and only if $\mathscr{S}\left(\alpha ; x_{0}\right)=\alpha\left(x_{0}\right)$.

3.10. Theorem. Let $X$ and $Y$ be spaces with $Y H$-closed and let $\alpha \in M(X, Y)$ have $u$-closed point images. Then $\alpha$ is w.u.s.c. at $x_{0} \in X$ if and only if $\mathscr{S}\left(\alpha ; x_{0}\right)=$ $\alpha\left(x_{0}\right)$.

Proof. Necessity. Since $\alpha\left(x_{0}\right)$ is $u$-closed, if $z \in Y-\alpha\left(x_{0}\right)$ there are sets $V \in \Sigma(z), W \in \Sigma\left(\alpha\left(x_{0}\right)\right)$ in $Y$ with $\operatorname{cl}(V) \cap \operatorname{cl}(W)=\varnothing$. There is a $Q \in \Sigma\left(x_{0}\right)$ in $X$ with $\alpha(Q) \subset \operatorname{cl}(W)$ since $\alpha$ is w.u.s.c. at $x_{0}$. $\operatorname{So} \operatorname{cl}(V) \cap \alpha(Q)=\varnothing$ and, consequently, $z \notin \mathscr{S}\left(\alpha ; x_{0}\right)$. Hence $\mathscr{S}\left(\alpha ; x_{0}\right) \subset \alpha\left(x_{0}\right)$ and $\mathscr{S}\left(\alpha ; x_{0}\right)=\alpha\left(x_{0}\right)$.

Sufficiency. If $W_{0} \in \Sigma\left(\alpha\left(x_{0}\right)\right)$ in $Y$ such that $\Omega=\left\{\alpha(V) \cap\left(Y-\operatorname{cl}\left(W_{0}\right)\right): V \in\right.$ $\left.\Sigma\left(x_{0}\right)\right\}$ is a filterbase on $Y$ then, since $Y$ is $H$-closed, we have $\varnothing \neq a d_{\theta} \Omega \cap$ $\operatorname{cl}\left(Y-\operatorname{cl}\left(W_{0}\right)\right) \subset \mathscr{S}\left(\alpha ; x_{0}\right)-W_{0}$. Thus $\alpha\left(x_{0}\right)-W_{0} \neq \varnothing$, a contradiction, and $\alpha$ is w.u.s.c. at $x_{0}$.

The proof of the theorem is complete.

3.11. Corollary. Let $X$ and $Y$ be spaces with $Y$ H-closed. Then a function $\alpha \in \mathcal{M}(X, Y)$ with $u$-closed values is weakly-continuous at $x_{0} \in X$ if and only if $\mathscr{S}\left(\alpha ; x_{0}\right)=\alpha\left(x_{0}\right)$.

The proof of Theorem 3.12 is omitted as it is similar to the proofs of the above theorems.

3.12. Theorem. Let $X$ and $Y$ be spaces with $Y \mathrm{H}$-closed and let $\alpha \in M(X, Y)$ have $u$-closed point images. Then $\alpha$ is $\theta$-u.s.c. at $x_{0} \in X$ if and only if $\mathscr{T}\left(\alpha ; x_{0}\right)=$ $\alpha\left(x_{0}\right)$.

Another of the main results of [3] (Theorem 3.3) states that $\mathscr{C}^{-1}(\alpha ; y)=$ $\operatorname{cl}\left(\alpha^{-1}(y)\right)$ for each $y \in Y$ when $X$ and $Y$ are spaces with $X$ regular and 
$\alpha \in M(X, Y)$ is a closed function. We employ the following definition from [11] to extend this result to multifunctions and to generalize this result as well as a result from [11].

3.13. Definition. If $X$ and $Y$ are spaces, $\alpha \in M(X, Y)$ is locally closed if for each $x \in X$ and for each neighborhood $V$ of $x$ there is a neighborhood $W$ of $x$ such that $W \subset V$ and $\alpha(W)$ is closed in $Y$.

We see easily that a closed multifunction defined on a regular space is locally closed. It is known that a closed multifunction need not be locally closed and that a locally closed multifunction need not be closed.

3.14. THEOREM. If $X$ and $Y$ are spaces and $\alpha \in M(X, Y)$ is locally closed then $\mathscr{C}^{-1}(\alpha ; y)=c \ell\left(\alpha^{-1}(y)\right)$ for each $y \in Y$.

Proof. We need show only that $\mathscr{C}^{-1}(\alpha ; y) \subset \operatorname{cl}\left(\alpha^{-1}(y)\right)$. Let $x \in$ $\mathscr{C}^{-1}(\alpha ; y)-\operatorname{cl}\left(\alpha^{-1}(y)\right)$, where $y \in Y$. There is a $V \in \Sigma(x)$ with $V \cap \alpha^{-1}(y)=\varnothing$. There is a $W \in \Sigma(x)$ with $\operatorname{cl}(\alpha(W)) \subset \alpha(V)$ since $\alpha$ is locally closed. So $y \notin \operatorname{cl}(\alpha(W))$ and there is a $Q \in \Sigma(y)$ in $Y$ with $Q \cap \alpha(W)=\varnothing$. Hence $W \cap \alpha^{-1}(Q)=\varnothing$; this is a contradiction and the proof is complete.

3.15. Corollary [11]. If $X$ and $Y$ are spaces and $\alpha \in M(X, Y)$ is locally closed with $\alpha^{-1}(y)$ closed for each $y \in Y$ then $\alpha$ has a closed graph.

The final result in this section improves upon Corollary 3.5 of [11] since $\boldsymbol{\theta}$-closure and closure coincide in regular spaces.

3.16. Theorem. Let $X$ and $Y$ be spaces and let $\alpha \in \mathcal{M}(X, Y)$ be closed with $\theta$-closed point inverses. Then $\alpha$ has a closed graph.

Proof. We show that $\mathscr{C}^{-1}(\alpha ; y) \subset \alpha^{-1}(y)$ for each $y \in Y$ (see Theorem 3.2). Assume that $x \in X, y \in Y$ and $x \notin \alpha^{-1}(y)$. Since $\alpha^{-1}(y)$ is $\theta$-closed there is a $V \in \Sigma(x)$ with $\operatorname{cl}(V) \cap \alpha^{-1}(y)=\varnothing$. So we have $\alpha(V) \subset \alpha(\operatorname{cl}(V)) \subset$ $\alpha\left(X-\alpha^{-1}(y)\right) \subset Y-\{y\}$. Since $\alpha(\operatorname{cl}(V))$ is closed we see that $y \notin \operatorname{cl}(\alpha(V))$ and, consequently, that $x \notin \mathscr{C}^{-1}(\alpha ; y)$. The proof is complete.

4. Multifunctions, inverse cluster sets, regularity and compactness generalizations. In this section we will characterize regularity, compactness, Lindelöfness, $m$-compactness and $H$-closedness in terms of multifunctions and inverse cluster sets. The first theorem characterizes regularity (not assuming $\left.T_{1}\right)$.

4.1. Theorem. A space $X$ is regular if and only if for each space $Y$, each closed $\alpha \in \mathcal{M}(X, Y)$ satisfies $\mathscr{C}^{-1}(\alpha ; y)={ }_{c} \ell\left(\alpha^{-1}(y)\right)$ for each $y \in Y$.

Proof. Necessity. A closed $\alpha \in M(X, Y)$ is locally closed when $X$ is regular. So the result of Theorem 3.14 holds. 
Sufficiency. Suppose $X$ satisfies the condition of the theorem and let $F \subset X$ be closed and nonempty. Choose $x_{0} \in F$ and define $\alpha \in M\left(X, X\left(x_{0}, \Sigma(F)\right)\right)$ by $\alpha(x)=x$ if $x \notin F$ and $\alpha(x)=\left\{x, x_{0}\right\}$ otherwise. Let $H \subset X$ be closed. If $H \cap F \neq \varnothing$, then $x_{0} \in \alpha(H)$ so $\alpha(H)$ is closed; since $H$ is closed in $X$, if $H \cap F=\varnothing$ there is a $V \in \Sigma(F)$ in $X$ with $V \cap H=\varnothing ; \alpha(V) \in \Sigma\left(x_{0}\right)$ in $X\left(x_{0}, \Sigma(F)\right)$ and $\alpha(V) \cap \alpha(H)=V \cap H=\varnothing$. Thus $\alpha(H)$ is closed in $X\left(x_{0}, \Sigma(F)\right)$. Thus by hypothesis we have $\mathscr{C}^{-1}\left(\alpha ; x_{0}\right)=\operatorname{cl}\left(\alpha^{-1}\left(x_{0}\right)\right)=F$; so if $y \notin F$ there is a $V \in \Sigma(y)$ in $X$ and a $W \in \Sigma\left(x_{0}\right)$ in $X\left(x_{0}, \Sigma(F)\right)$ with $V \cap \alpha^{-1}(W)=\varnothing$. Since $\alpha^{-1}(W)=W$ and $W \in \Sigma(F)$ in $X$ the sufficiency is proved.

The proof of the theorem is complete.

Before presenting the other theorems in this section we present some preliminary definitions and known results. A subset $K$ of a space $X$ is $m$-compact for an infinite cardinal $m$ if each closed filterbase $\Omega$ on $K$ with cardinality at most $m$ satisfies $K \cap a d \Omega \neq \varnothing$. The following theorems (4.2-4.5) appear in [5].

4.2. Theorem. A subset $K$ of a space is m-compact if and only if each filterbase $\Omega$ on the space such that $\Omega$ has at most $M$ elements and such that $F \cap V \neq \varnothing$ is satisfied for each $F \in \Omega$ and $V \in \Sigma(K)$ satisfies $K \cap$ ad $\Omega \neq \varnothing$.

4.3. Theorem, A subset $K$ of a space is Lindelöf if and only if each filterbase $\Omega$ on the space such that $I \cap V \neq \varnothing$ is satisfied by each $V \in \Sigma(K)$ and countable intersection, $I$, of elements of $\Omega$ satisfies $K \cap$ ad $\Omega \neq \varnothing$.

4.4 TheOrem. A subset $K$ of a space $X$ is quasi $H$-closed relative to $X$ if and only if each filterbase $\Omega$ on $X$ such that $F \cap C \neq \varnothing$ is satisfied for each $F \in \Omega$ and $C \in \nabla(K)$ satisfies $K \cap a d_{\theta} \Omega \neq \varnothing$.

4.5. Theorem. If $X$ is quasi $H$-closed and $K \subset X$ then $c \ell_{\theta}(K)$ is quasi $H$-closed relative to $X$.

We recall that a space is a $\mathscr{P}$-space in the sense of Gillman and Jerison [1] if each $G_{\delta}$ is open. If $\boldsymbol{m}$ is an infinite cardinal we say that a space $X$ has character $m$ if at each point there is a local open base of cardinality at most $m$. We will have use for the following notation.

4.6 Notation. If $X$ and $Y$ are spaces, $K \subset Y$ and $\alpha \in M(X, Y)$, we use the notation (a) $\mathscr{C}^{-1}(\alpha ; K)=\bigcup_{K} \mathscr{C}^{-1}(\alpha ; y)$, (b) $\mathscr{S}^{-1}(\alpha ; K)=\bigcup_{K} \mathscr{S}^{-1}(\alpha ; y)$ and (c) $\mathscr{T}^{-1}(\alpha ; K)=\bigcup_{K} \mathscr{T}^{-1}(\alpha ; y)$.

4.7. TheOREM. Let $X$ be a space with character $m$ and let $Y$ be any space. If $\alpha \in \mathcal{M}(X, Y)$ and $K \subset Y$ is $m$-compact then $\mathscr{C}^{-1}(\alpha, K)=\bigcap_{\Sigma(K)} c \ell\left(\alpha^{-1}(V)\right)$. 
Proof. For each $y \in K$ we have $\Sigma(K) \subset \Sigma(y)$, so $\mathscr{C}^{-1}(\alpha ; y)=$ $\bigcap_{\Sigma(y)} \operatorname{cl}\left(\alpha^{-1}(V)\right) \subset \bigcap_{\Sigma(K)} \operatorname{cl}\left(\alpha^{-1}(V)\right) \quad$ and, consequently, $\quad \mathscr{C}^{-1}(\alpha ; K) \subset$ $\bigcap_{\Sigma(K)} \operatorname{cl}\left(\alpha^{-1}(V)\right)$. Now, let $z \in \bigcap_{\Sigma(K)} \operatorname{cl}\left(\alpha^{-1}(V)\right)$ and let $\Delta$ be a local base at $z$ of cardinality at most $m$. Then for each $W \in \Delta$ and $V \in \Sigma(K)$ in $Y$ we have $\alpha(W) \cap V \neq \varnothing$. So $\alpha(\Delta)$ is a filterbase on $Y$ which along with the $m$-compact subset $K$ satisfies the hypothesis of Theorem 4.2. Hence $K \cap \operatorname{ad} \alpha(\Delta) \neq \varnothing$. For each $y \in K \cap a d \alpha(\Delta)$ we have $V \cap \alpha(W) \neq \varnothing$ and, consequently, $W \cap$ $\alpha^{-1}(V) \neq \varnothing$ for each $V \in \Sigma(y)$ in $Y$ and $W \in \Delta$. Thus $z \in \mathscr{C}^{-1}(\alpha ; y)$ and the proof is complete.

Corollary 4.8 is a consequence of Theorem 4.7 and Theorem 3.2.

4.8. Corollary. Let $X$ be a space with character $m$ and let $Y$ be any space. If $\alpha \in M(X, Y)$ has a closed graph then $\alpha^{-1}(K)$ is closed in $X$ for each $m$-compact $K \subset Y$.

Proof. $\mathscr{C}^{-1}(\alpha ; K)=\bigcup_{K} \mathscr{C}^{-1}(\alpha ; y)=\bigcup_{K} \alpha^{-1}(y)=\alpha^{-1}(K)$. The proof is complete.

4.9. TheOREM. The following statements are equivalent for a $T_{1}$ space $Y$ :

(a) $Y$ is $m$-compact.

(b) $\mathscr{C}^{-1}(\alpha ; K)=\bigcap_{\Sigma(K)} c \ell\left(\alpha^{-1}(V)\right)$ for each space $X$ with character $m, \alpha \in$ $M(X, Y)$ and closed subset $K \subset Y$.

(c) $\mathscr{C}^{-1}(\alpha ; K)$ is closed in $X$ for each space $X$ with character $m, \alpha \in \mathcal{M}(X, Y)$ and closed subset $K \subset Y$.

Proof that (a) implies (b). This follows from Theorem 4.7 and the fact that a closed subset of an $m$-compact space is $m$-compact.

Proof that (b) implies (c). Obvious.

Proof that (c) implies (a). Let $\Omega$ be a filterbase on $Y$ with cardinality at most $m$ and let $y_{0} \in Y$ with $a d \Omega \cap\left(Y-\left\{y_{0}\right\}\right)=\varnothing$. Let $\alpha \in \mathcal{M}\left(Y\left(y_{0}, \Omega\right), Y\right)$ be the identity function. We readily observe that $Y\left(y_{0}, \Omega\right)$ has character $m$. Now let $V \in \Sigma\left(y_{0}\right)$ in $Y$. Then $\mathscr{C}^{-1}(\alpha ; Y-V) \supset Y-V$ and is closed in $Y\left(y_{0}, \Omega\right)$ by hypothesis. If $y \in Y-V$ then $y \notin a d \Omega$ and $y \neq y_{0}$; so, since $Y$ is $T_{1}$, there is an $F \in \Omega$ and a $W \in \Sigma(y)-\Sigma\left(y_{0}\right)$ in $Y$ satisfying $W \cap F=\varnothing$. Thus $y_{0} \notin \mathscr{C}^{-1}(\alpha ; y)$. Consequently, $y_{0} \notin \operatorname{cl}(Y-V)$ in $Y\left(y_{0}, \Omega\right)$ and there is an $F \in \Omega$ satisfying $F \subset V$. The proof of (c) implies (a) is complete.

The proof of the theorem is complete.

4.10. Theorem. Let $X$ be a $\mathscr{P}$-space and let $Y$ be any space. If $\alpha \in \mathcal{M}(X, Y)$ and $K \subset Y$ is Lindelöf then $\mathscr{C}^{-1}(\alpha ; K)=\bigcap_{\Sigma(K)} c \ell\left(\alpha^{-1}(V)\right)$.

Proof. We know that $\mathscr{C}^{-1}(\alpha ; K) \subset \bigcap_{\Sigma(K)} \operatorname{cl}\left(\alpha^{-1}(V)\right)$ for any spaces $X, Y$, subset $K \subset Y$, and $\alpha \in M(X, Y)$ (see proof of Theorem 4.7). Now, let $z \in$ $\bigcap_{\Sigma(K)} \operatorname{cl}\left(\alpha^{-1}(V)\right)$. Then $\alpha(\Sigma(z))$ is a filterbase on $Y$ which along with the Lindelöf 
subset $K$ satisfies the hypothesis of Theorem 4.3. The proof is completed as in the proof of Theorem 4.7.

Corollary 4.11 is a consequence of Theorem 4.10 and Theorem 3.2.

4.11. Corollary. Let $X$ be a $\mathscr{P}$-space and let $Y$ be any space. If $\alpha \in$ $M(X, Y)$ has a closed graph then $\alpha^{-1}(K)$ is closed in $X$ for each Lindelöf $K \subset Y$.

4.12. TheOREM. The following statements are equivalent for a $T_{1}$ space $Y$ :

(a) $Y$ is Lindelöf.

(b) $\mathscr{C}^{-1}(\alpha ; K)=\bigcap_{\Sigma(K)} c \ell\left(\alpha^{-1}(V)\right)$ for each $\mathscr{P}$-space $X, \alpha \in M(X, Y)$ and closed subset $K \subset Y$.

(c) $\mathscr{C}^{-1}(\alpha ; K)$ is closed in $X$ for each $\mathscr{P}$-space $X, \alpha \in \mathcal{M}(X, Y)$ and closed subset $K \subset Y$.

Proof. We prove only that (c) implies (a) . Let $\Omega$ be a filterbase on $Y$ with the countable intersection property and assume, without loss, that $\Omega$ is closed under countable intersection. This proof is completed as in the proof of (c) implies (a) of Theorem 4.9 if we observe that $Y\left(y_{0}, \Omega\right)$ is a $\mathscr{P}$-space.

The final result in this section is a characterization of $H$-closed spaces in terms of inverse cluster sets.

4.13. Theorem. A Hausdorf space $Y$ is $H$-closed if and only if $\bigcap_{\nabla\left(c \ell_{\theta}(K)\right)} c \ell_{\theta}\left(\alpha^{-1}(C)\right) \subset \mathscr{T}^{-1}\left(\alpha ;{ }_{c} \ell_{\theta}(K)\right)$ for each space $X, \alpha \in M(X, Y)$ and $K \subset Y$.

Proof. Necessity. If $Y$ is $H$-closed, $X$ is any space, $K \subset Y$ and $\alpha \in M(X, Y)$, then for each $x \in \bigcap_{\nabla\left(\mathrm{cl}_{\theta}(K)\right)} \mathrm{cl}_{\theta}\left(\alpha^{-1}(C)\right), \alpha(\Gamma(x))$ is a filterbase on $Y$ which along with $\operatorname{cl}_{\theta}(K)$ (quasi $H$-closed relative to $X$ by Theorem 4.5) satisfies the hypothesis of Theorem 4.4. So $\operatorname{cl}_{\theta}(K) \cap a d_{\theta} \alpha(\Gamma(x)) \neq \varnothing$. For each $y \in$ $\operatorname{cl}_{\theta}(K) \cap a d_{\theta} \alpha(\Gamma(x))$ we have $x \in \mathscr{T}^{-1}(\alpha ; y)$. The proof of the necessity is complete.

Sufficiency. Let $\Omega$ be a filterbase on $Y$ and let $y_{0} \in Y$ with $\operatorname{ad}_{\theta} \Omega \cap(Y-$ $\left.\left\{y_{0}\right\}\right)=\varnothing$. Let $\alpha \in M\left(Y\left(y_{0}, \Omega\right), Y\right)$ be the identity function and let $V \in \Sigma\left(y_{0}\right)$ in $Y$. If $K=Y-\operatorname{cl}(V)$ and $y \in \operatorname{cl}_{\theta}(K)$ then $y \neq y_{0}$ and $y \notin a d_{\theta} \Omega$; since $Y$ is $T_{2}$ there is an $F \in \Omega$ and a $W \in \Sigma(y)-\Sigma\left(y_{0}\right)$ in $Y$ satisfying $\operatorname{cl}(W) \cap F=\varnothing$. So $y_{0} \notin \mathscr{T}^{-1}(\alpha ; y)$. This means that $y_{0} \notin \bigcap_{\nabla\left(\mathrm{cl}_{\theta}(K)\right)} \mathrm{cl}_{\theta}\left(\alpha^{-1}(C)\right)$. So $y_{0} \notin \operatorname{cl}(Y-\operatorname{cl}(V))$ in $Y\left(y_{0}, \Omega\right)$ and there is an $F \in \Omega$ satisfying $F \subset \operatorname{cl}(V)$.

The proof of the theorem is complete.

5. Inverse cluster sets, connected and connectivity multifunctions. In this section we extend some results from [3] and [7] for connected and connectivity functions. If $X$ and $Y$ are sets and $\alpha \in M(X, Y)$ we let $\lambda_{\alpha} \in M(X, X \times Y)$ be the multifunction defined by $\lambda_{\alpha}(x)=\{x\} \times \alpha(x)$. We will call $\alpha$ a connected multifunction if $\alpha$ preserves connected sets, and a connectivity multifunction if $\lambda_{\alpha}$ is connected. Theorem 5.1 extends Theorem 4.1 of [3] to multifunctions. 
5.1. Theorem. Let $X$ and $Y$ be spaces with $X$ compact and let $\alpha \in M(X, Y)$ be connected with compact point images. Then $\alpha$ is a connectivity multifunction if for each connected $M \subset X$ and $x \in M, \mathscr{C}^{-1}(\alpha ; \alpha(x)) \cap_{c} \ell(M)=\{x\}$.

Proof. Let $\alpha$ be connected and assume the given condition. Suppose there is a connected $M \subset X$ with $\lambda_{\alpha}(M)=H \cup K$ where $H$ and $K$ are separated and let $A=\lambda_{\alpha}^{-1}(H) \cap M$ and $B=\lambda_{\alpha}^{-1}(K) \cap M$. If $x \in A$ then $\lambda_{\alpha}(x)$ is connected as the product of connected sets; $\lambda_{\alpha}(x) \cap H \neq \varnothing$ and $\lambda_{\alpha}(x) \subset H \cup K$ so $\lambda_{\alpha}(x) \subset H$. Since $\alpha(x)$ is compact and $\lambda_{\alpha}(x) \cap \operatorname{cl}(K)=\varnothing$ there are sets $V \in \Sigma(x)$ in $X$ and $W \in \Sigma(\alpha(x))$ in $Y$ with $(V \times W) \cap K=\varnothing$. If, moreover, $a \in V \cap B$ we have $\lambda_{\alpha}(a)$ connected, $\lambda_{\alpha}(a) \subset \lambda_{\alpha}(M)$ and $\lambda_{\alpha}(a) \cap K \neq \varnothing$; so $\lambda_{\alpha}(a) \subset K$ and this establishes that $\alpha(V \cap B) \cap W=\varnothing$. Furthermore, $\alpha(M)=\alpha(A) \cup \alpha(B)$, $\alpha(A) \neq \varnothing$ and $\alpha(B) \neq \varnothing$. Suppose $y \in \operatorname{cl}(\alpha(B)) \cap \alpha(x)$ where $x \in A$. Then $\Omega=$ $\left\{\alpha^{-1}(Q) \cap B: Q \in \Sigma(y)\right\}$ is a filterbase on $\operatorname{cl}(M)$, which is compact. Thus ad $\Omega \cap$ $\operatorname{cl}(M) \neq \varnothing$. If $v \in a d \Omega \cap \operatorname{cl}(M)$ then $v \in \mathscr{C}^{-1}(\alpha ; y) \subset \mathscr{C}^{-1}(\alpha ; \alpha(x))$. Also, for the sets $W \in \Sigma(y)$ and $V \in \Sigma(x)$ above we have $\alpha(V \cap B) \cap W \neq \varnothing$; so $V \cap$ $\left(B \cap \alpha^{-1}(W)\right)=\varnothing$ and, consequently, $v \neq x$. However, $\{v, x\} \subset \mathscr{C}^{-1}(\alpha ; \alpha(x)) \cap$ $\operatorname{cl}(M)$. This is a contradiction, so $\alpha(A) \cap \operatorname{cl}(\alpha(B))=\varnothing$. Similarly, $\alpha(B) \cap$ $\operatorname{cl}(\alpha(A))=\varnothing$. This means that $\alpha(M)$ is pot connected. With this contradiction the proof is complete.

Our next and final theorem extends Theorems 3.1 and 3.2 of [7].

5.2. Theorem. Let $X$ and $Y$ be spaces and let $\alpha \in M(X, Y)$ be connected and have closed point images. Then $c \ell(F) \subset \alpha^{-1}(c \ell(\alpha(F)))$ for each connected $F \subset X$.

Proof. Let $x \in \operatorname{cl}(F)$. Then $F \cup\{x\}$ is connected in $X$, so $\alpha(F \cup\{x\})=$ $\alpha(F) \cup \alpha(x)$ and, consequently, $\operatorname{cl}(\alpha(F)) \cup \alpha(x)$ is connected in $Y$. Since $\operatorname{cl}(\alpha(F))$ and $\alpha(x)$ are both closed in $Y$ we must have $\operatorname{cl}(\alpha(F)) \cap \alpha(x) \neq \varnothing$. The proof is complete.

5.3 Remark. Since the preparation of this paper Theorem 5.1 has been published as the main result in [12]. The proof given above is different and shorter than that given in [12].

\section{REFERENCES}

1. L. Gillman and M. Jerison, Rings of continuous functions, Van Nostrand, New York, 1960.

2. L. L. Herrington, Remarks on $H(i)$ spaces and strongly-closed graphs, Proc. Amer. Math. Soc., 58 (1976), 277-283.

3. T. R. Hamlett and P. E. Long, Inverse cluster sets, Proc. Amer. Math. Soc., 53 (1975), 470-476.

4. L. L. Herrington and P. E. Long, Characterizations of H-closed spaces, Proc. Amer. Math. Soc., 48 (1975), 469-475.

5. J. E. Joseph, Multifunctions and cluster sets, Proc. Amer. Math. Soc., 74, (1979) 329-337.

6. N. Levine, A decomposition of continuity in topological spaces, Amer. Math. Monthly 68 (1961), 44-46. 
7. W. J. Pervin and N. Levine, Connected mappings of Hausdorff spaces, Proc. Amer. Math. Soc., 9 (1958), 488-496.

8. J. Porter and J. Thomas, On H-closed and minimal Hausdroffl spaces, Trans. Amer. Math. Soc., 138 (1969), 159-170.

9. R. E. Smithson, Multifunctions, Nieuw Archief voor Wiskunde (3) 20 (1972), 31-53.

10. R. E. Smithson, Almost and weak continuity for multifunctions, Preprint.

11. R. E. Smithson, Subcontinuity for multifunctions, Pacific J. Math. 61 (1975), 283-288.

12 R. E. Smithson, Connected and connectivity multifunctions, Proc. Amer. Math. Soc., 64 (1977), 146-148

13. M. K. Singal and A. R. Singal, Almost continuous mappings, Yokohama Math. J., 16 (1968), 63-73.

14. N. V. Veličko, H-closed topological spaces, Mat. Sb., 70 (112) (1966), 98-112; Amer. Math. Soc. Transl., 78 (Series 2) (1969), 103-118.

\section{DePARTMENT OF MATHEMATICS \\ HOWARD UNIVERSITY}

WASHINGTON, D.C. 20059 